\title{
Suppression of Mutual Interference in Noncontiguous Orthogonal Frequency Division Multiplexing Based Cognitive Radio Systems
}

\author{
Atif Elahi, ${ }^{1}$ Ijaz Mansoor Qureshi, ${ }^{2}$ Fawad Zaman, ${ }^{3}$ Noor Gul, ${ }^{1}$ and Tahir Saleem ${ }^{1}$ \\ ${ }^{1}$ Department of Electrical Engineering, Faculty of Engineering and Technology, International Islamic University, Islamabad, Pakistan \\ ${ }^{2}$ Department of Electrical Engineering, Air University, Islamabad, Pakistan \\ ${ }^{3}$ Department of Electrical Engineering, COMSATS Institute of Information Technology, Attock Campus, Attock, Pakistan \\ Correspondence should be addressed to Atif Elahi; atif.phdee40@iiu.edu.pk
}

Received 26 June 2016; Revised 9 November 2016; Accepted 16 November 2016; Published 16 January 2017

Academic Editor: Rafael Pérez-Jiménez

Copyright (C) 2017 Atif Elahi et al. This is an open access article distributed under the Creative Commons Attribution License, which permits unrestricted use, distribution, and reproduction in any medium, provided the original work is properly cited.

\begin{abstract}
Orthogonal frequency division multiplexing (OFDM) is a favourable technology for dynamic spectrum access (DSA) due to the flexibility in spectrum shaping. In spite of that, high sidelobes of OFDM subcarriers bring in considerable interference to the nearby users, particularly in OFDM based cognitive radio (CR) networks, where the secondary users (SUs) are capable of accessing the spectrum opportunistically. In this paper, two new techniques for the suppression of high sidelobes are proposed. The proposed techniques composed of an optimization scheme are followed by generalized sidelobe canceller. The proposed techniques can be considered as a two-level suppression technique in the sense that in the first level the sidelobe is reduced by using cancellation carriers (CCs), whose amplitudes are determined using genetic algorithm (GA) and differential evolution (DE), while in the second level further reduction of sidelobe is achieved using generalized sidelobe canceller (GSC). Simulation results show the power spectral density (PSD) performance of the proposed techniques in comparison with already existing techniques, demonstrating that the proposed techniques minimize the out-of-band radiation (OOBR) significantly, thus qualifying for more effective spectrum sharing.
\end{abstract}

\section{Introduction}

To tackle the problem of spectrum overcrowding, Mitola [1$3]$ was the first to give the concept of cognitive radio (CR). The philosophy of CR is to search for an inactive spectrum at a certain time and localities, dynamically, and try to use them in such a way that the secondary user cannot interfere with the licensed users (LUs). Orthogonal frequency division multiplexing (OFDM), considered as the perfect candidate for CR [4-6], can divide the total available bandwidth into a number of orthogonal subbands and deactivate specific subcarriers.

Some advantages of OFDM include spectral efficiency, resistivity against frequency selective fading, protection against interference, and simpler channel equalization. However, due to the high sidelobes of the OFDM subcarriers, CR based OFDM experience high out-of-band radiation
(OOBR) that may interfere with either LUs or secondary users (SUs) in the contiguous bands. Different techniques including cancellation carriers (CCs) $[7,8]$, CC using genetic algorithm (GA) and differential evolution (DE) [9], active and null cancellation carriers (ANCC) [10], advanced cancellation carriers (ACC) [11], advanced subcarrier weightings (ASW) [12], subcarrier weightings (SW) [13], filtering [14], windowing $[15,16]$, adaptive symbol transition (AST) [17], active interference cancellation (AIC) [18-20], insertion of guard bands [21, 22], multiple choice sequence (MCS) [23], spectral precoding schemes [24-28], constellation expansion (CE) [29-31], and sidelobes reduction using generalized sidelobe canceller (GSC) [32] are proposed to address the OOBR problem. These techniques undergo different penalties; therefore, proper hybridization of two or more techniques may result in a better OOBR reduction. 
In this paper, we propose two new techniques: GSC combined with CC using GA and GSC combined with CC using DE. In these new techniques, the sidelobes are reduced into two steps. In the first step, CCs are added to the original OFDM signal, where the amplitudes of CCs have been adjusted using GA and DE [9], respectively, while in the second step the signal is then passed through GSC $[32,33]$ for further reduction of sidelobes.

The remainder of the paper is organized as follows. In Section 2, we discuss the system model. In Section 3, a brief discussion of our proposed techniques is given. Section 4 is about the simulation results, while in Section 5 conclusion about the paper is given.

Notation. $(\cdot)^{H},(\cdot)^{T}$, and $(\cdot)^{-1}$ represent the Hermitian, transpose, and inverse, while $E[\cdot]$ represents the expectation. Small bold letter represents a vector, the capital bold letter represents matrix, and I represents the identity matrix while $\mathrm{O}$ represents the null matrix.

\section{System Model}

Consider an OFDM system having a total of $M$ secondary users (SUs) opportunistically using a spectrum not utilized by a $L U$, which is identified with the help of spectrum sensing techniques. The total available spectrum is divided into $N$ subcarriers out of which $N_{s}$ subcarriers are utilized by ith SU, such that $N_{s} \subseteq N$. The baseband OFDM signal for $i$ th SU is given by

$$
x_{i}(t)=\sum_{n=0}^{N_{s}-1} q_{n, i} e^{j 2 \pi f_{n} t} I(t)
$$

where $x_{i}(t)$ represents the OFDM signal of $i$ th $\mathrm{SU}, q_{n, i}$ represents the modulated symbol on $n$th subcarrier, and $f_{n}=$ $n / T, n=0,1, \ldots, N_{s}$, represents the subcarrier frequencies, while $I(t)$ represents the rectangular function defined as

$$
I(t)= \begin{cases}1 & -T_{g} \leq t \leq T \\ 0 & \text { otherwise }\end{cases}
$$

where $T_{g}$ represents the guard interval length used for the elimination of intersymbol interference (ISI) and $T$ represents the symbol duration.

After taking the Fourier transformation of the signal given in (1), we get

$$
X(f)=\sum_{n=0}^{N_{s}-1} q_{n, i} \sin c\left(\pi\left(f-f_{n}\right) T_{s}\right),
$$

where $T_{s}=T+T_{g}$ represents the symbol duration, while $\sin c(x)=\sin (x) /(x)$.

The signal in (3) is SU signal in frequency domain, whose sidelobe power in frequency bands of the adjacent LUs decays with $1 / f^{2} N_{s}$ as shown in Figure 1, resulting in high OOBR. To protect those adjacent LUs from the OOBR of SUs, the sidelobes should be suppressed efficiently.

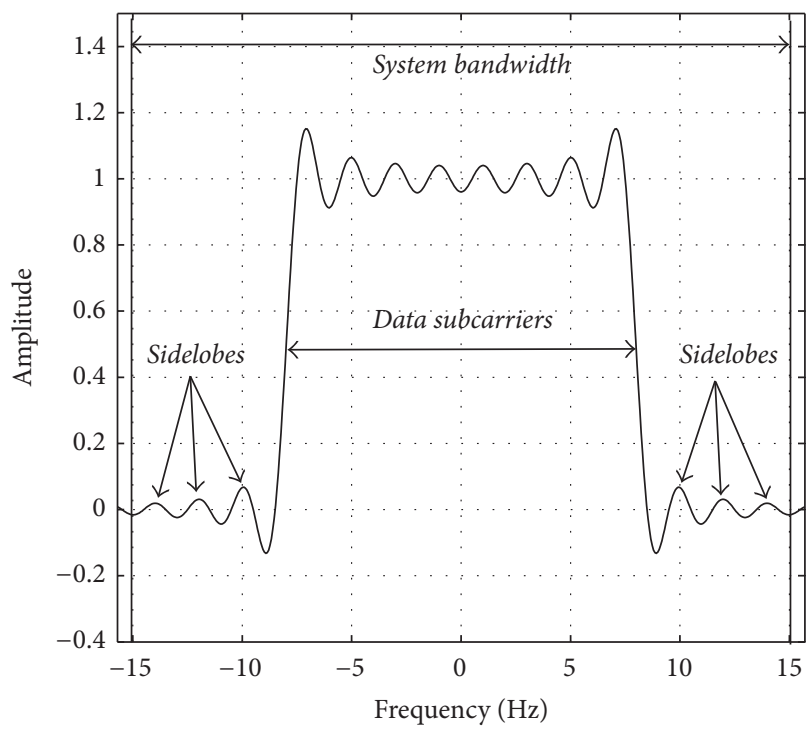

FIGURE 1: Spectrum of transmitted OFDM signal.

\section{The Proposed Methodologies}

In this section, we present our two new techniques for suppression of sidelobes. The proposed technique I includes a combination of GSC with CC using GA, while the proposed technique II includes a combination of GSC with CC using DE. In both of these proposed techniques, the sidelobe suppression is done in two steps.

3.1. Step I. The first step involves the suppression of sidelobes using CCs, whose amplitudes are calculated using GA in our first proposed methodology and using DE in our second proposed methodology. The concept of GA was first given by Holland [34] in the early 1970s and in late 1980s by Goldberg [35]. The main steps of GA in the form of pseudocode are given as follows:

\section{Start}

Randomly generate a set of candidate solutions (i.e., set of chromosomes).

Compute the fitness of each candidate solution in the current population.

Repeat

Select the parents in the order of their fitness.

Produce the offspring using crossover (single point crossover, multiple point crossover).

Offspring for the new generation is selected using one of three methods, that is, Elitism. Replace generation and survival of fitness. If no improvement is found in the new generation, then do mutation. 
All the offspring will be the new population; the parents will die.

Until a stop condition is satisfied.

\section{End.}

The basic idea behind GA comes from Darwin's theory of evolution, randomly generating a set of "chromosomes" and then allowing for the process of "natural selection" and "genetic drift" to occur to develop the "chromosome" into the most appropriate contestant for "survival." Every "chromosome" is the only probable solution of the problem being solved, and the "natural selection" is a method of crossover where some qualities of better performing "chromosomes" are combined with the qualities of other "chromosomes" for the production of the next generation. Next is the "genetic drift," a kind of mutation of a "chromosome" that is usually represented by a probability which dictates the chance of random mutation in the form of inversion of a bit or a similar random change to the "chromosome." GA has the capability of finding a good solution to a problem where the iterative solution takes too much time and its solution is not achievable. Another significance of using GA is that there are many problems with many different constraints based on the specific of the solution for which one is searching. Another strength of genetic algorithm's approach is that there exists a proof of convergence for an elitist version of the genetic algorithm. Although the theory of the GA is not very complex, the single parameters and execution of the GA generally need a large amount of tuning.

The concept of DE was first given by Stone and Price $[36,37]$. The technique of DE is the same as GA; it can be applied to real-valued problems over a continuous space with much more simplicity than a GA. The idea behind the method of $\mathrm{DE}$ is that the difference between two vectors yields a difference vector which can be used with a scaling factor to traverse the search space. The procedural step of DE in the form of pseudocode is given as follows:

Start

Randomly generate the initial population. Compute the fitness of the initial population. Repeat

Select three different solutions randomly. Create one offspring using the DE operators.

Do this a number of times equal to the population size.

For each member of the next generation, if $\operatorname{off} \operatorname{spring}(x)$ is more fit than parent $(x)$, Parent $(x)$ is replaced.

Until a stop condition is satisfied.

End.

The strength of DE methodology is that it regularly shows better results than GA and other evolutionary algorithms

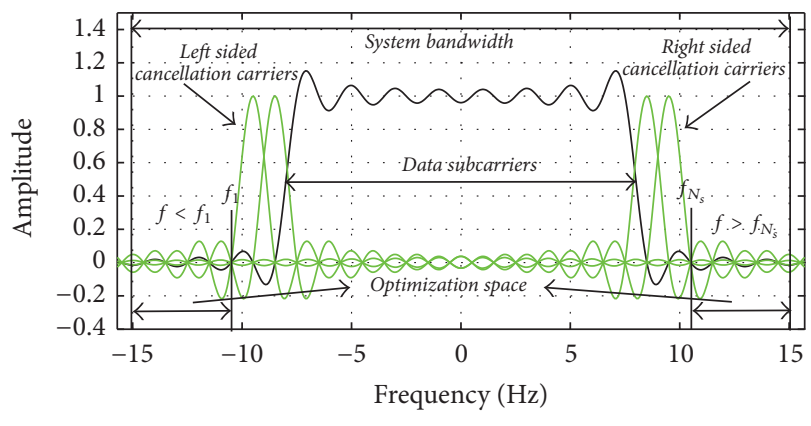

FIGURE 2: Concept of cancellation carriers.

(EAs). DE is simple and straightforward and can be easily applied to a widespread range of real-valued problems in spite of noisy, multimodal, multidimensional spaces, which usually make the problems very difficult for optimization. Another extraordinary feature of DE is that the parameters such as crossover rate and mutation do not need the same fine tuning which is required in many other EAs.

3.1.1. Concept of Cancellation Carriers. As discussed earlier, the total number of available subcarriers is $N$, out of which $N_{s}$ subcarriers are used by the $i$ th SU for its data transmission and carry modulated symbols $q_{n, i} \in \mathbf{q}_{\mathbf{i}}=\left[q_{1, i}, q_{2, i}, \ldots, q_{N_{s}, i}\right]^{T}$. The remaining $N-N_{s}$ subcarriers are not used for data transmission but act like guard carriers. As an alternative of guard carriers, $K=K_{l}+K_{r}$ CCs are inserted on either side of the used OFDM signal of $i$ th SU as shown in Figure 2, where $K_{l}$ and $K_{r}$ represent left and right sided CCs. These CCs are not used to carry the data but complex weights, that is, the amplitude of main lobe of CCs $a_{k} \in \mathbf{a}=\left[a_{1}, a_{2}, \ldots, a_{K}\right]^{T}$ that should be adjusted in such a way that their sidelobes cancel the sidelobes of the OFDM signal of the $i$ th SU in a certain defined space called optimization space. The transmitted symbol vector now consists of $N_{s}$ data symbols and $K$ weights of CCs and is normalized in such a way that the power of transmitted OFDM signal of $i$ th SU with CCs is the same as without CCs. The spectrum of the $k$ th $\mathrm{CC}$ is given by

$$
c_{k}(\alpha)=a_{k} \sin c\left(\pi\left(\alpha-\lambda_{k}\right)\right), \quad k=1,2, \ldots, K
$$

where $\alpha=\left(f-f_{o}\right) T_{s}$, while $\lambda_{k}$ representing the normalized centre frequency of the $k$ th CC that lies on either side of the OFDM spectrum of $i$ th SU is given as

$$
\lambda_{k}=\left\{\begin{array}{cc}
\left(f_{1-k}-f_{o}\right) T_{s} & k=1,2, \ldots, K_{l} \\
\left(f_{N_{s}+k-K_{l}}-f_{o}\right) T_{s} & k=K_{l}+1, \ldots, K
\end{array}\right\} .
$$


Now, the transmitted OFDM signal of $i$ th SU with CCs in frequency domain is given by

$$
\begin{aligned}
& T(f)=\left(\sum_{k=0}^{K / 2-1} a_{k} \sin c\left(\pi\left(\alpha-\lambda_{k}\right)\right)\right. \\
& +\sum_{n=0}^{N_{s}-1} q_{n, i} \sin c\left(\pi\left(f-f_{n}\right) T_{s}\right)+\sum_{k=K / 2}^{K-1} a_{k} \\
& \left.\cdot \sin c\left(\pi\left(\alpha-\lambda_{k}\right)\right)\right) .
\end{aligned}
$$

To calculate the weights $a_{k} \in \mathbf{a}=\left[a_{1}, a_{2}, \ldots, a_{K}\right]^{T}$ of the CCs, GA and DE have been proposed which are based on our fitness function.

3.1.2. Fitness Function. The concept behind our proposed fitness function is that weights of CCs are calculated using the average of all samples. These samples are taken as the centre of each sidelobe for reducing the computational complexity and reduction of memory usage. Suppose the total number of samples taken on both sides of the OFDM signal is $M$. The result of the final fitness function is the average sidelobe power value of all samples given by

$$
\begin{aligned}
& A_{C j}=\left(\sum_{m_{1}=0}^{K / 2-1} A_{m_{1}, j} a_{m_{1}}+\sum_{m_{2}=K / 2}^{K-1} A_{m_{2}, j} a_{m_{2}}\right), \\
& A_{D j}=\left(\sum_{n=0}^{N_{s}-1} A_{n, j} d_{i, n}\right), \quad j=1,2, \ldots, M .
\end{aligned}
$$

In (7), $A_{m_{1}, j}$ represents the sidelobe level of $m_{1}$ th left CC at the $j$ th sample, $A_{m_{2}, i}$ represents the sidelobe level of $m_{2}$ th right CC at the $j$ th sample, and $a_{m_{1}}$ and $a_{m_{2}}$ represent the weights of the main lobe of the $m_{1}$ th left and $m_{2}$ th right CC. In (8), $A_{n, j}$ represents the sidelobe power level of $n$th data subcarrier at $j$ th sample and $d_{i, n}$ represents the weight of the main lobe of $n$th data subcarrier. Finally, the total sidelobe power level at $j$ th sample from the data subcarriers and CCs is given by

$$
A_{j}=A_{D j}+A_{C j} .
$$

On putting the values of $A_{D j}$ and $A_{C j}$ from (7) and (8) in (9), we obtain the following:

$$
\begin{aligned}
A_{j} & =\left(\sum_{m_{1}=0}^{K / 2-1} A_{m_{1}, j} a_{m_{1}}+\sum_{n=0}^{N_{s}-1} A_{n, j} d_{i, n}\right. \\
& \left.+\sum_{m_{2}=K / 2}^{K-1} A_{m_{2}, j} a_{m_{2}}\right) .
\end{aligned}
$$

The final fitness will now become

$$
A=\left(\frac{1}{M} \sum_{j=1}^{M} A_{j}\right) .
$$

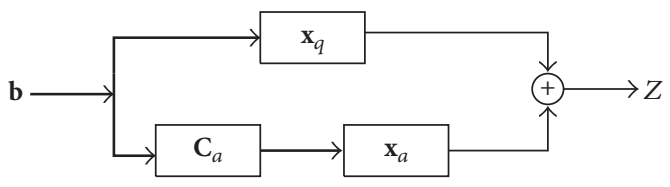

FIgURE 3: Block diagram of GSC.

3.2. Step II. In the second step, the samples of the signal with optimized CCs given in (6) are collected into a vector $\mathbf{b}=\left[b_{0}, b_{1}, \ldots, b_{W-1}\right]^{T}$, having dimension $W \times 1$ whose elements are uncorrelated. The vector $\mathbf{b}$ is taken to the GSC for further suppression of sidelobes. GSC is a substitute to express the notion that the linearly constrained minimum variance (LCMV) problem can provide an understanding, is very effective for investigation, and can make the LCMV beamforming easier. It also provides the relationship between the multiple sidelobe canceller (MSC) and LCMV beamforming. GSC is a method of converting the constrained minimization problem into an unconstrained one. The block diagram of GSC is shown in Figure 3, which consists of two portions: the upper portion and the lower portion. The upper portion is the main part of GSC, which consists of quiescent weight vector $\mathbf{x}_{q}$ that maintains the signal of the SU that is here in this case the signal of $i$ th SU, and gives an essential gain to the desired part of the signal that is the part of the signal from $f_{1}$ to $f_{N_{s}}$ fulfilling the constraints as depicted in Figure 2.

The lower portion of the GSC comprises blocking matrix $\mathbf{C}_{a}$ which stops the desired part of the signal and keeps the sidelobes, that is, $f<f_{1}$ and $f>f_{N_{s}}$, portion of the signal depicted in Figure 2 and an adaptive weight vector $\mathbf{x}_{a}$ that alters the weights of sidelobes. Finally, the signals from the upper portion and lower portion are subtracted from each other, resulting in the further reduction of sidelobes.

The output of GSC after passing vector $\mathbf{b}$ is given by [32, 33]

$$
Z=\mathbf{x}^{H} \mathbf{b},
$$

where $\mathbf{x}^{H}=\left(\mathbf{x}_{q}^{H}-\mathbf{x}_{a}^{H} \mathbf{C}_{a}^{H}\right)$ and its dimension is $1 \times W$.

The weight vector $\mathbf{x}^{H}=\left(\mathbf{x}_{q}^{H}-\mathbf{x}_{a}^{H} \mathbf{C}_{a}^{H}\right)$ that minimizes the output power of (12) with multiple linear constraints using LCMV is given by

$$
\begin{array}{ll}
\min _{\mathbf{x}} & \mathbf{x}^{H} \mathbf{R x}, \\
\text { s.t. } & \mathbf{x}^{H} \mathbf{C}=\mathbf{f}^{H} .
\end{array}
$$

Its solution is given by

$$
\mathbf{x}_{o}^{H}=\mathbf{f}^{H}\left(\mathbf{C}^{H} \mathbf{R C}\right)^{-1} \mathbf{C}^{H} \mathbf{R}^{-1},
$$

where

$$
\mathbf{R}=E\left[\mathbf{b b}^{H}\right]=\left[\begin{array}{ccc}
\sigma_{\mathbf{b}}^{2} & \ldots & 0 \\
\vdots & \ddots & \vdots \\
0 & \ldots & \sigma_{\mathbf{b}}^{2}
\end{array}\right]
$$


represents the correlation matrix having dimension $W \times W$, $\sigma_{\mathbf{b}}^{2}$ represents the variance, and $\mathbf{C}$ represents the $W \times N_{s}$ constraint matrix, having $N_{s}$ steering vectors given by

$$
\mathbf{C}=\left[\mathbf{s}_{1}, \mathbf{s}_{2}, \ldots, \mathbf{s}_{N_{s}}\right],
$$

where $N_{s}$ represents the frequency number in the defined portion of the signal shown in Figure 2. $\mathbf{s}_{j}=\left[s_{1}, s_{2}, \ldots, s_{W}\right]^{T}$ is a $j$ th steering vector having dimension $W \times 1$, which contains $W$ samples of the $j$ th spectrum, while $\mathbf{f}=[1,1, \ldots, 1]^{T}$ representing the gain vector having dimension $N_{s} \times 1$ contains gain associated with each steering vector.

The useful implementation of LCMV is the division of $W$-dimensional space into constraint subspace defined by columns of $\mathbf{C}$ and orthogonal subspace defined by columns of $\mathbf{C}_{a}$. Thus,

$$
\mathbf{C}^{H} \mathbf{C}_{a}=\mathbf{O},
$$

where $\mathbf{O}$ is the null matrix having dimension $N \times\left(W-N_{s}\right)$ and $\mathbf{C}_{a}$ is the blocking matrix having dimension $W \times\left(W-N_{s}\right)$.

Now, consider the partition of $\mathbf{x}_{o}^{H}$ into two orthogonal components

$$
\mathbf{x}_{o}^{H}=\mathbf{x}_{c}^{H}-\mathbf{x}_{P}^{H},
$$

where $\mathbf{x}_{c}^{H}$ is the projection of $\mathbf{x}_{o}^{H}$ onto the constraint subspace and $\mathbf{x}_{P}^{H}$ is the projection of $\mathbf{x}_{o}^{H}$ onto the orthogonal subspace. The projection matrices onto the constraint and orthogonal subspace both having dimension $W \times W$ are given by

$$
\begin{aligned}
& \mathbf{P}_{c}=\mathbf{C}\left(\mathbf{C}^{H} \mathbf{C}\right)^{-1} \mathbf{C}^{H}, \\
& \mathbf{P}_{o}=\mathbf{C}_{a}\left(\mathbf{C}_{a}^{H} \mathbf{C}_{a}\right)^{-1} \mathbf{C}_{a}^{H} .
\end{aligned}
$$

So, therefore,

$$
\begin{aligned}
& \mathbf{x}_{c}^{H}=\mathbf{x}_{o}^{H} \mathbf{P}_{c}, \\
& \mathbf{x}_{P}^{H}=\mathbf{x}_{o}^{H} \mathbf{P}_{o} .
\end{aligned}
$$

On putting (14) and (19) into (21), we get

$$
\mathbf{x}_{c}^{H}=\mathbf{f}^{H}\left(\mathbf{C}^{H} \mathbf{C}\right)^{-1} \mathbf{C}^{H} \triangleq \mathbf{x}_{q}^{H}
$$

defined as the quiescent weight vector with dimension $1 \times W$. Also, putting (14) and (20) into (22) gives

$$
\mathbf{x}_{P}^{H}=\mathbf{f}^{H}\left(\mathbf{C}^{H} \mathbf{R}^{-1} \mathbf{C}\right)^{-1} \mathbf{C}^{H} \mathbf{R}^{-1} \mathbf{C}_{a}\left(\mathbf{C}_{a}^{H} \mathbf{C}_{a}\right)^{-1} \mathbf{C}_{a}^{H} .
$$

Equation (24) is correct but is not particularly useful, so it is suggested to be divided into two portions. The first portion contains $\mathbf{C}_{a}$, the blocking matrix, while the second portion contains $\mathbf{x}_{a}$, the adaptive weight vector having dimension $\left(W-N_{s}\right) \times 1$. For obtaining the blocking matrix $\mathbf{C}_{a}$, we will first find $\mathbf{P}_{o}$ using

$$
\mathbf{P}_{o}=\mathbf{I}-\mathbf{P}_{c}
$$

and orthonormalizing $\mathbf{P}_{o}$ and then taking the first $W-N_{s}$ columns of the orthonormalized matrix, resulting in the blocking matrix $\mathbf{C}_{a}$ with the following property:

$$
\mathbf{C}_{a}^{H} \mathbf{C}_{a}=\mathbf{I} \text {. }
$$

For adaptive weight vector $\mathbf{x}_{a}$ substituting $\mathbf{x}^{H}=\left(\mathbf{x}_{q}^{H}-\mathbf{x}_{a}^{H} \mathbf{C}_{a}^{H}\right)$ in (12),

$$
Z=\left(\mathbf{x}_{q}^{H}-\mathbf{x}_{a}^{H} \mathbf{C}_{a}^{H}\right) \mathbf{b} .
$$

The power output is

$$
P=\left(\mathbf{x}_{q}^{H}-\mathbf{x}_{q}^{H} \mathbf{C}_{q}^{H}\right) \mathbf{R}\left(\mathbf{x}_{q}-\mathbf{C}_{a} \mathbf{x}_{a}\right) .
$$

Taking gradient of (28) with respect to $\mathbf{x}_{a}$ and setting the results equals to zero, we get an optimum adaptive weight vector $\widehat{\mathbf{x}}_{a}^{H}$ given by

$$
\widehat{\mathbf{x}}_{a}^{H}=\mathbf{x}_{q}^{H} \mathbf{R} \mathbf{C}_{a}\left(\mathbf{C}_{a}^{H} \mathbf{R} \mathbf{C}_{a}\right)^{-1} .
$$

\section{Simulation Results}

In this section, we consider five different spectrum sharing scenarios to check the efficiency and authenticity of our proposed techniques. We compare the performance of our proposed techniques with the current techniques with the help of computer simulations in terms of normalized power spectral density (PSD). As discussed above, DE is simple and straightforward to implement and has much better performance in terms of accuracy, convergence speed, computational complexity, and robustness as compared to the other EAs like GA and others. Therefore, the performance of proposed technique II is better as compared to the performance of proposed technique I in all five different spectrum sharing scenarios.

4.1. Scenario I. In this scenario, assume that $\mathrm{CR}$ detects a single vacant band divided into 16 OFDM subcarriers mapped with BPSK and is utilized by a single SU. The efficiency of our proposed techniques for this scenario is compared with the current techniques, including CC $[7,8]$, ACC [11], ASW [12], CC using GA and DE [9], and GSC [32], as shown in Figures 4 and 5. In all simulated results, a total of four CCs are considered. From Figures 4 and 5 , it is observed that the proposed techniques give better suppression of sidelobes as compared with other techniques.

4.2. Scenario II. In this scenario, assume that CR detects four vacant bands, denoted by II, IV, VI, and VIII, all having equal bandwidths. The spacing between these bands denoted by I, III, V, VII, and IX is also considered as of equal bandwidths. SUs functioning in bands II, IV, VI, and VIII use 32 OFDM subcarriers, mapped with BPSK. The performances of the proposed techniques in terms of PSD with others, including ASW [12], ACC [11], CC [7, 8], CC using GA and DE [9], and GSC [32], are shown in Figures 6 and 7. Two CCs on either side of the original spectrum are inserted in all the CC based suppression techniques. Figures 6 and 7 show that the proposed technique got a reduction considerably, as compared with other existing techniques. 


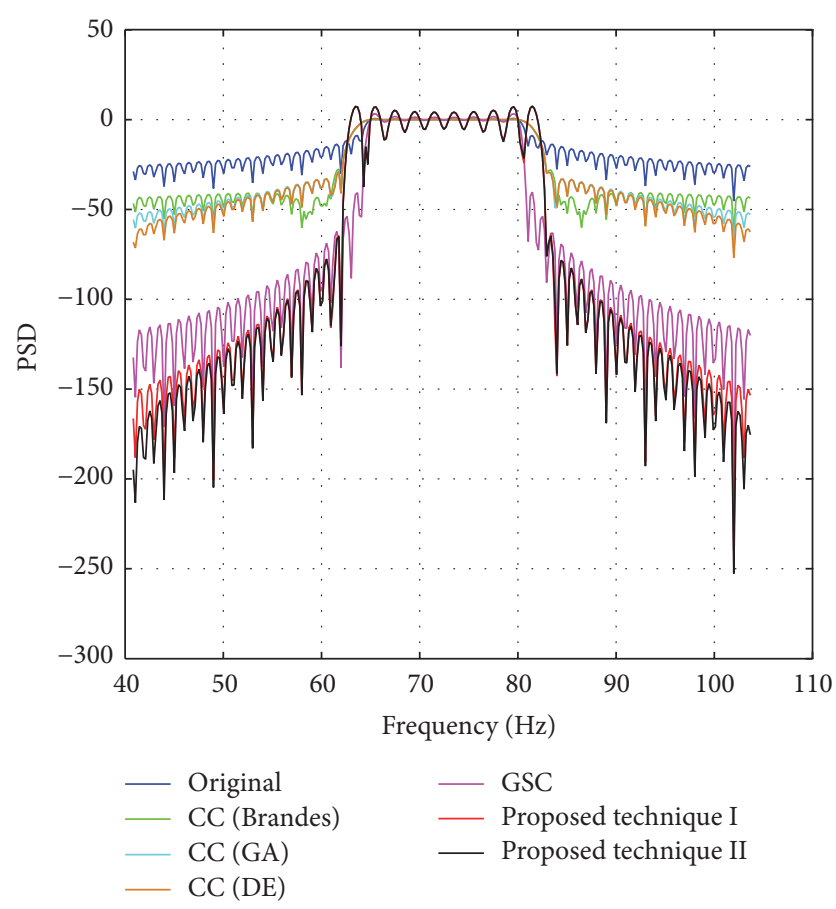

FIGURE 4: The PSD performance comparison between the proposed techniques and existing techniques, Scenario I.

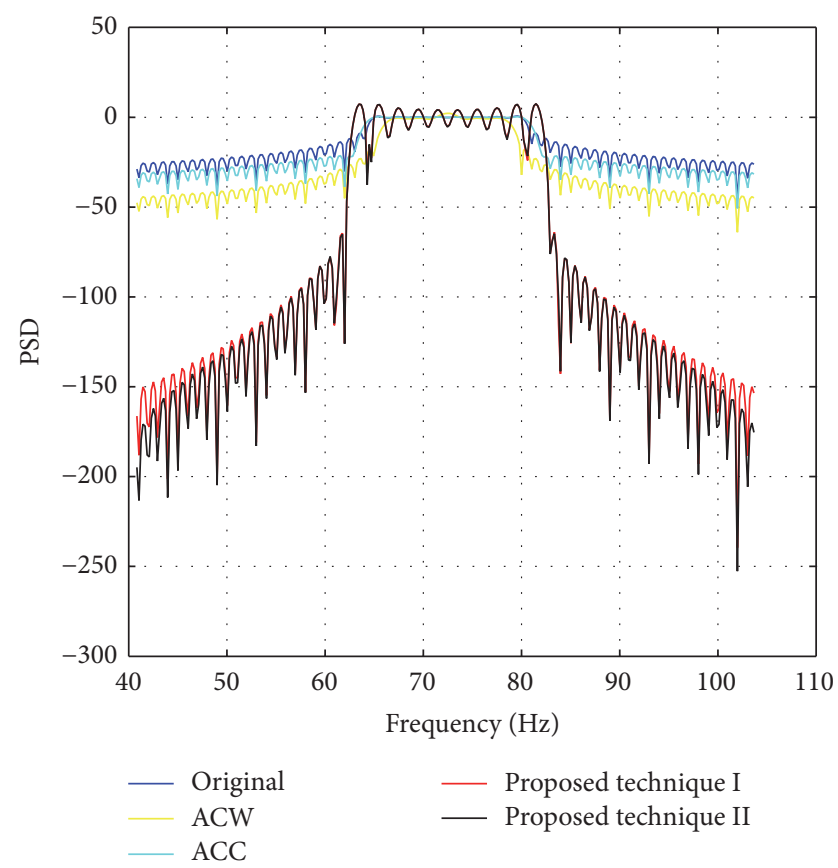

FIGURE 5: The PSD performance comparison between the proposed techniques and existing techniques, Scenario I.

4.3. Scenario III. In this scenario, consider the notion that white holes detected by CR are given by II, IV, VI, and VIII, which have the same bandwidth. The spacing between white holes is given by I, III, V, VII, and IX, respectively, which have different bandwidths. An equal number of OFDM subcarriers (i.e., 32) are used by the SUs operating in these white holes,

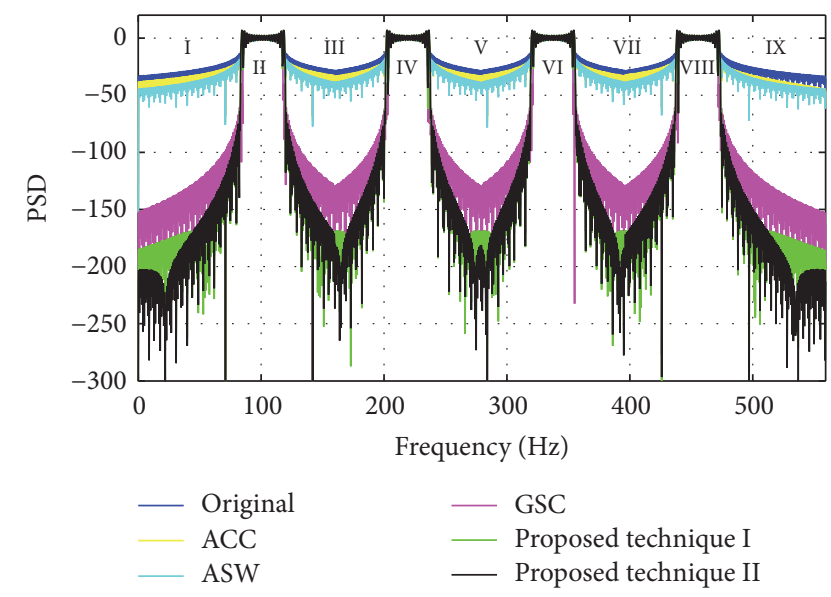

FIGURE 6: The PSD performance comparison between the proposed techniques and existing techniques, Scenario II.

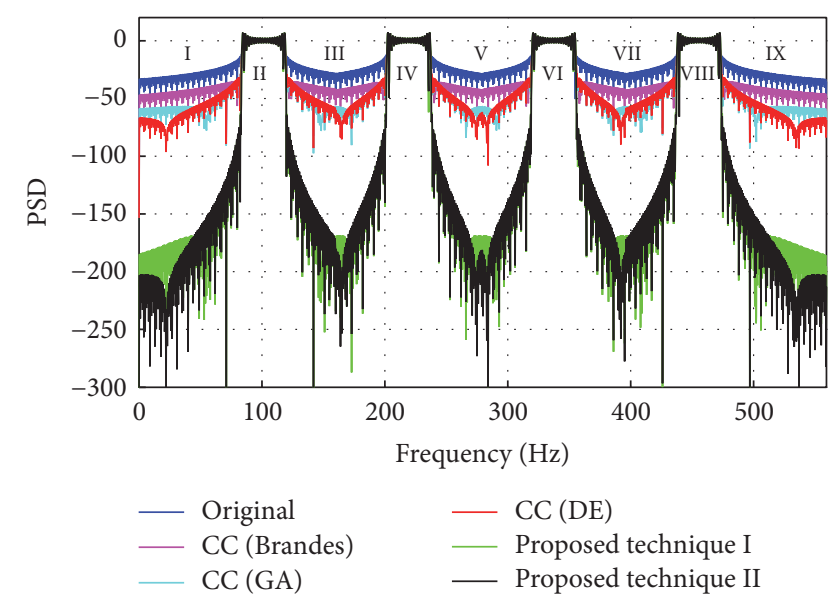

FIGURE 7: The PSD performance comparison between the proposed techniques and existing techniques, Scenario II.

mapped with BPSK. The reliability and effectiveness of the proposed techniques are shown in Figures 8 and 9 that show the comparison in terms of PSD with current techniques, including CC using GA and DE [9], ASW [12], ACC [11], CC $[7,8]$, and GSC [32]. The proposed techniques get better suppression in that spectrum sharing scenario and outclass all the existing techniques.

4.4. Scenario $I V$. In this scenario, the bandwidth of the spectral white holes detected by CR is considered as unequal represented as II, IV, VI, and VIII. The spacing between them is considered as equal. SUs operating in white hole II use 16 subcarriers, in IV use 32, in VI use 64, and in VIII use 128 , each modulated with BPSK. Figures 10 and 11 show that the proposed techniques are effective and reliable in such a spectrum sharing scenario and get significant reduction of sidelobes in comparison with the current techniques 


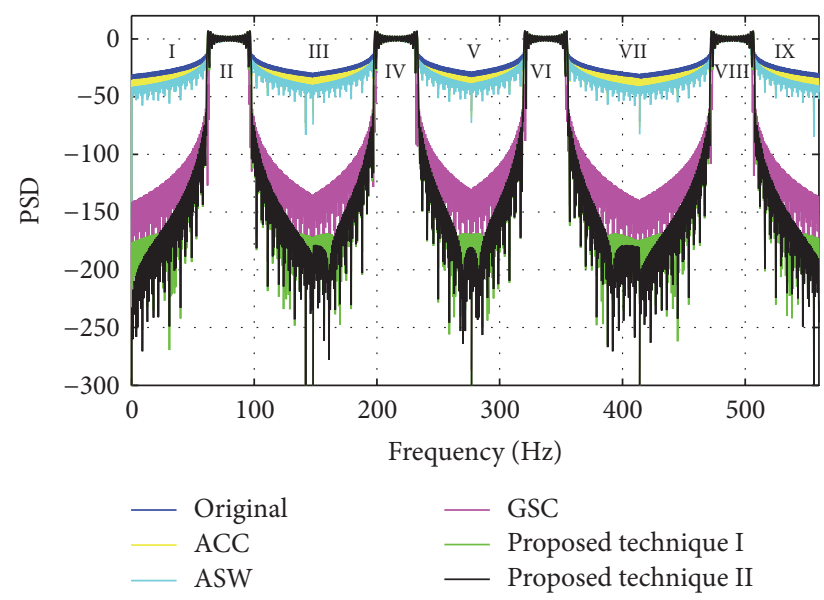

FIGURE 8: The PSD performance comparison between the proposed techniques and existing techniques, Scenario III.

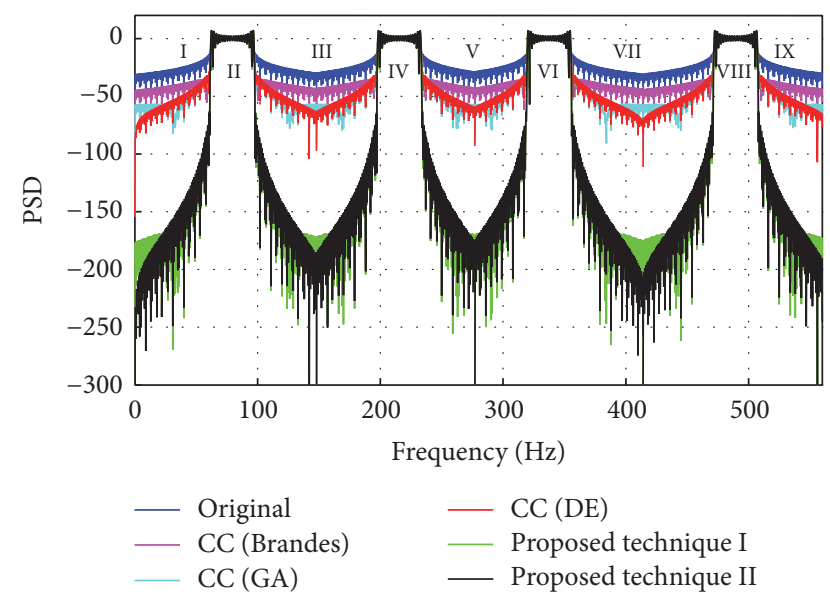

FIGURE 9: The PSD performance comparison between the proposed techniques and existing techniques, Scenario III.

including CC using GA and DE [9], ASW [12], ACC [11], CC $[7,8]$, and GSC [32].

4.5. Scenario $V$. In this scenario, four spectral white spaces are considered to be detected by CR, represented by II, IV, VI, and VIII, which have unequal bandwidth. Spacing between them is also considered to be unequal. SUs operating in these spectral white spaces use 16 subcarriers in II, 32 in IV, 64 in VI, and 128 in VIII, modulated with BPSK. The performances of our proposed techniques for that scenario compared with the current techniques including CC [7, 8], CC using GA and DE [9], ACC [11], ASW [12], and GSC [32] are given in Figures 12 and 13, which shows that the proposed scheme outclasses all the existing techniques and gets significant reduction.

\section{Conclusion}

In this paper, the OOBR problem, one of the major issues in $\mathrm{CR}$ based OFDM, is discussed. To handle that problem, we

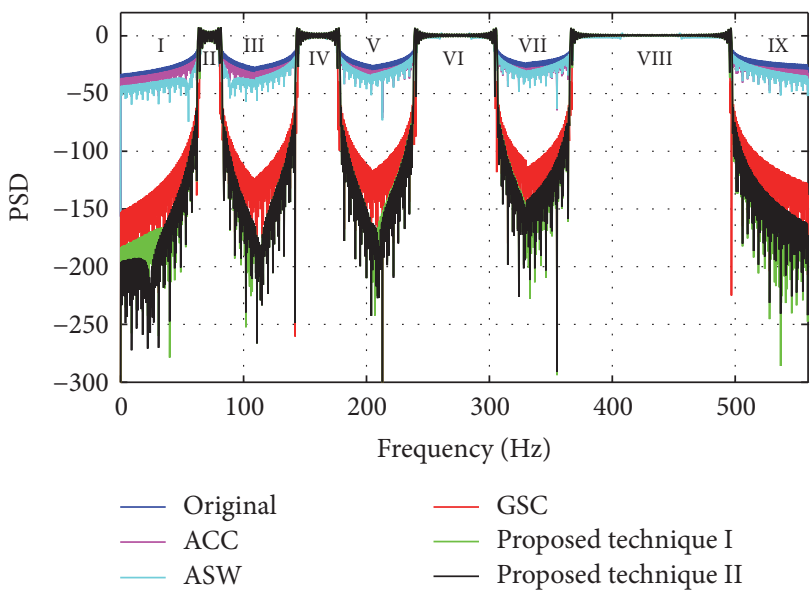

FIGURE 10: The PSD performance comparison between the proposed techniques and existing techniques, Scenario IV.

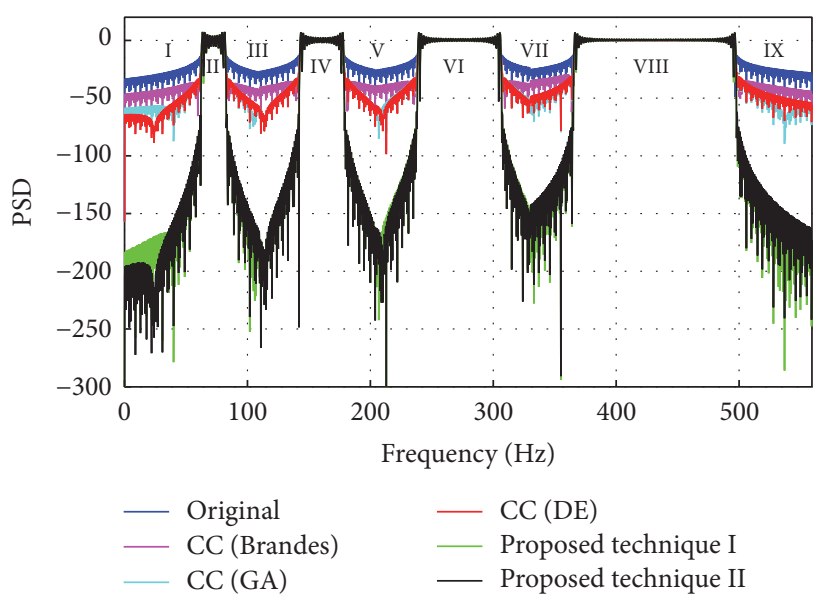

FIGURE 11: The PSD performance comparison between the proposed techniques and existing techniques, Scenario IV.

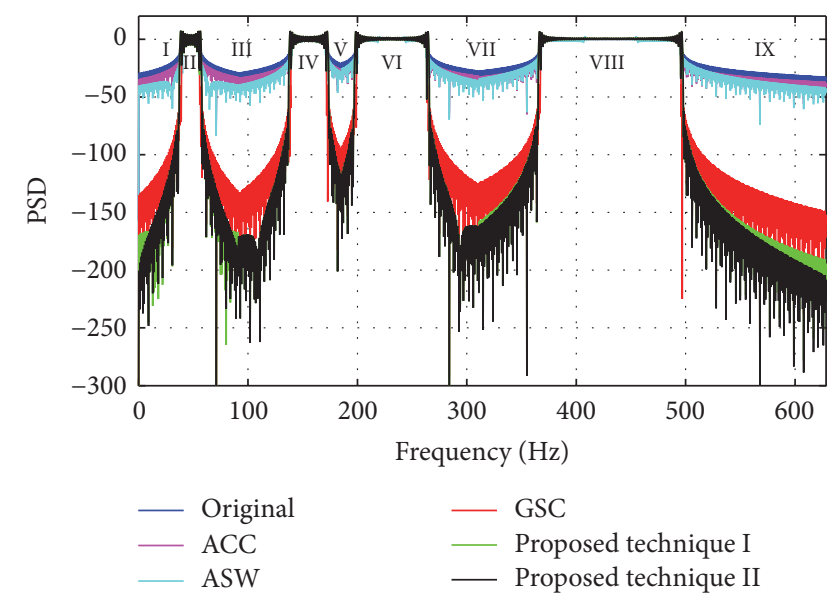

FIGURE 12: The PSD performance comparison between the proposed techniques and existing techniques, Scenario V. 


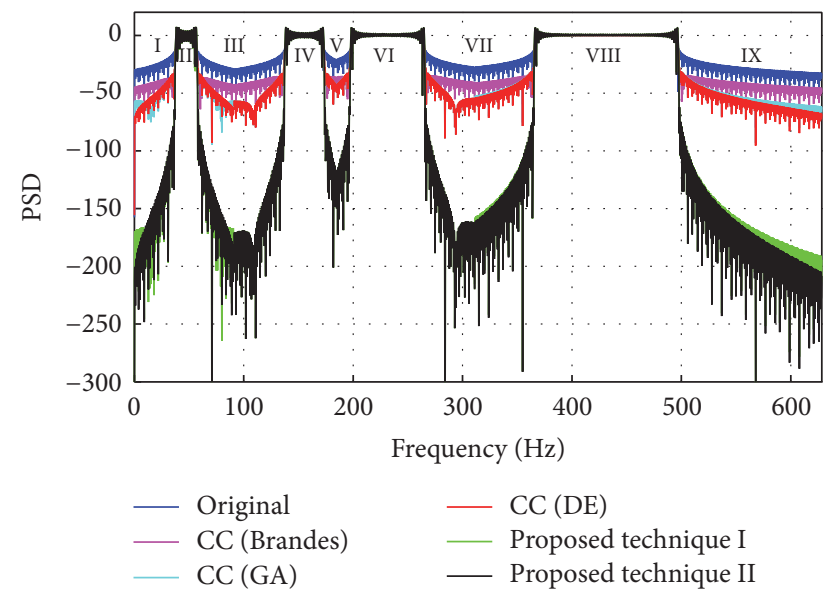

FIGURE 13: The PSD performance comparison between the proposed techniques and existing techniques, Scenario V.

propose two new techniques: the first one is the combination of CCs using GA with GSC and the second one is the combination of CCs using DE with GSC. The purpose of combining different techniques is to take advantage of the individual techniques for a better reduction of OOBR. The strength and reliability of the proposed techniques are shown via computer simulations in different types of spectrum sharing scenarios, which shows that the proposed techniques get far better reduction of OOBR as compared to the existing techniques and the overall performance of the proposed technique is better.

\section{Competing Interests}

The authors declare that there are no competing interests regarding the publication of this paper.

\section{References}

[1] T. A. Weiss and F. K. Jondral, "Spectrum pooling: an innovative strategy for the enhancement of spectrum efficiency," IEEE Communications Magazine, vol. 42, no. 3, pp. S8-S14, 2004.

[2] J. Mitola III and G. Q. Maguire Jr., "Cognitive radio: making software radios more personal," IEEE Personal Communications, vol. 6, no. 4, pp. 13-18, 1999.

[3] F. Meucci, O. Cabral, F. J. Velez, A. Mihovska, and N. R. Prasad, "Spectrum aggregation with multi-band user allocation over two frequency bands," in Proceedings of the IEEE Mobile WiMAX Symposium (MWS '09), pp. 81-86, Napa Valley, Calif, USA, July 2009.

[4] H. Mahmoud, T. Yücek, and H. Arslan, "OFDM for cognitive radio: merits and challenges," IEEE Wireless Communications, vol. 16, no. 2, pp. 6-15, 2009.

[5] Y. Zhang and C. Leung, "Resource allocation in an OFDMbased cognitive radio system," IEEE Transactions on Communications, vol. 57, no. 7, pp. 1928-1931, 2009.

[6] S. Dikmese, A. Loulou, S. Srinivasan, and M. Renfors, "Spectrum sensing and resource allocation models for enhanced
OFDM based cognitive radio," in Proceedings of the 9th International Conference on Cognitive Radio Oriented Wireless Networks (CROWNCOM '14), pp. 360-365, Oulu, Finland, June 2014.

[7] S. Brandes, I. Cosovic, and M. Schnell, "Sidelobe suppression in OFDM systems by insertion of cancellation carriers," in Proceedings of the 62nd Vehicular Technology Conference (VTC '05), pp. 152-156, IEEE, Dallas, Tex, USA, September 2005.

[8] S. Pagadarai, A. M. Wyglinski, and R. Rajbanshi, "A sub-optimal sidelobe suppression technique for OFDM-based cognitive radios," in Proceedings of the IEEE Military Communications Conference (MILCOM '08), pp. 1-6, San Diego, Calif, USA, November 2008.

[9] A. Elahi, I. M. Qureshi, F. Zaman, and F. Munir, "Reduction of out of band radiation in non-contiguous OFDM based cognitive radio system using heuristic techniques," Journal of Information Science and Engineering, vol. 32, pp. 349-364, 2016.

[10] F. R. B. Lopes and J. S. G. Panaro, “OFDM sidelobe suppression combining active and null cancellation carriers in the guard bands," in Proceedings of the 15th SBMO/IEEE MTT-S International Microwave \& Optoelectronics Conference (IMOC '13), pp. 1-5, IEEE, Rio de Janeiro, Brazil, August 2013.

[11] A. Selim, I. Macaluso, and L. Doyle, "Efficient sidelobe suppression for OFDM systems using advanced cancellation carriers," in Proceedings of the IEEE International Conference on Communications (ICC '13), pp. 4687-4692, IEEE, Budapest, Hungary, June 2013.

[12] A. Selim and L. Doyle, "Real-time sidelobe suppression for OFDM systems using advanced subcarrier weighting," in Proceedings of the IEEE Wireless Communications and Networking Conference (WCNC '13), pp. 4043-4047, IEEE, Shanghai, China, April 2013.

[13] I. Cosovic, S. Brandes, and M. Schnell, "Subcarrier weighting: a method for sidelobe suppression in OFDM systems," IEEE Communications Letters, vol. 10, no. 6, pp. 444-446, 2006.

[14] D. Noguet, M. Gautier, and V. Berg, "Advances in opportunistic radio technologies for TVWS," EURASIP Journal on Wireless Communications and Networking, vol. 2011, article 170, 2011.

[15] M. S. El-Saadany, A. F. Shalash, and M. Abdallah, "Revisiting active cancellation carriers for shaping the spectrum of OFDMbased cognitive radios," in Proceedings of the IEEE Sarnoff Symposium (SARNOFF '09), pp. 1-5, IEEE, Princeton, NJ, USA, April 2009.

[16] A. Sahin and H. Arslan, "Edge windowing for OFDM based systems," IEEE Communications Letters, vol. 15, no. 11, pp. 12081211, 2011.

[17] H. A. Mahmoud and H. Arslan, "Sidelobe suppression in OFDM-based spectrum sharing systems using adaptive symbol transition," IEEE Communications Letters, vol. 12, no. 2, pp. 133134, 2008.

[18] H. Yamaguchi, "Active interference cancellation technique for MB-OFDM cognitive radio," in Proceedings of the Conference Proceedings- 34th European Microwave Conference, pp. 11051108, The Hague, Netherlands, October 2004.

[19] Z. Wang, D. Qu, T. Jiang, and Y. He, "Spectral sculpting for OFDM based opportunistic spectrum access by extended active interference cancellation," in Proceedings of the IEEE Global Telecommunications Conference (GLOBECOM '08), pp. 44424446, December 2008.

[20] D. Qu, Z. Wang, and T. Jiang, "Extended active interference cancellation for sidelobe suppression in cognitive radio OFDM 
systems with cyclic prefix," IEEE Transactions on Vehicular Technology, vol. 59, no. 4, pp. 1689-1695, 2010.

[21] T. Weiss, J. Hillenbrand, A. Krohn, and F. K. Jondral, "Mutual interference in OFDM-based spectrum pooling systems," in Proceedings of the IEEE 59th Vehicular Technology Conference (VTC-Spring '04), vol. 4, pp. 1873-1877, IEEE, Milan, Italy, May 2004.

[22] P. Layer, "Specifications: high-speed physical layer in the $5 \mathrm{GHz}$ band," IEEE Std 802, 1999.

[23] I. Cosovic and T. Mazzoni, "Suppression of sidelobes in OFDM systems by multiple-choice sequences," European Transactions on Telecommunications, vol. 17, no. 6, pp. 623-630, 2006.

[24] J. van de Beek, "Orthogonal multiplexing in a subspace of frequency well-localized signals," IEEE Communications Letters, vol. 14, no. 10, pp. 882-884, 2010.

[25] M. Ma, X. Huang, B. Jiao, and Y. J. Guo, "Optimal orthogonal precoding for power leakage suppression in DFT-based systems," IEEE Transactions on Communications, vol. 59, no. 3, pp. 844-853, 2011.

[26] J. Van De Beek and F. Berggren, "N-continuous OFDM," IEEE Communications Letters, vol. 13, no. 1, pp. 1-3, 2009.

[27] C.-D. Chung, “Spectrally precoded OFDM," IEEE Transactions on Communications, vol. 54, no. 12, pp. 2173-2185, 2006.

[28] Z. You, J. Fang, and I.-T. Lu, "Combination of spectral and SVD precodings for out-of-band leakage suppression," in Proceedings of the IEEE Long Island Systems, Applications and Technology Conference (LISAT '13), pp. 1-6, IEEE, Farmingdale, NY, USA, May 2013.

[29] D. Li, X. Dai, and H. Zhang, "Sidelobe suppression in NCOFDM systems using constellation adjustment," IEEE Communications Letters, vol. 13, no. 5, pp. 327-329, 2009.

[30] S. Pagadarai, R. Rajbanshi, A. M. Wyglinski, and G. J. Minden, "Sidelobe suppression for OFDM-based cognitive radios using constellation expansion," in Proceedings of the IEEE Wireless Communications and Networking Conference (WCNC '08), pp. 888-893, April 2008.

[31] A. Selim, B. Ozgul, and L. Doyle, "Efficient sidelobe suppression for OFDM systems with peak-to-average power ratio reduction," in Proceedings of the IEEE International Symposium on Dynamic Spectrum Access Networks (DYSPAN '12), pp. 510-516, Bellevue, Wash, USA, October 2012.

[32] A. Elahi, I. M. Qureshi, Z. U. Khan, and F. Zaman, "Sidelobe reduction in non-contiguous OFDM-based cognitive radio systems using a generalized sidelobe canceller," Applied Sciences, vol. 5, pp. 894-909, 2015.

[33] S. A. Hussain, T. Shah, N. A. Azam, A. A. D. Andrade, and A. Naveed Malik, "Spectrum distribution in cognitive radio: error correcting codes perspective," International Journal of Distributed Sensor Networks, vol. 10, no. 7, Article ID 864916, 2014.

[34] J. H. Holland, Adaptation in Natural and Artificial Systems: An Introductory Analysis with Applications to Biology, Control, and Artificial Intelligence, U Michigan Press, 1975.

[35] D. E. Goldberg, Genetic Algorithms in Search Optimization and Machine Learning, Addison-Wesley, Boston, Mass, USA, 1993.

[36] R. Storn and K. Price, "Differential evolution-a simple and efficient heuristic for global optimization over continuous spaces," Journal of Global Optimization, vol. 11, no. 4, pp. 341359, 1997.

[37] K. Price, R. M. Storn, and J. A. Lampinen, Differential Evolution: A Practical Approach to Global Optimization, Springer Science \& Business Media, 2006. 


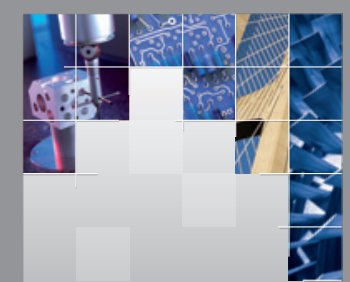

\section{Enfincering}
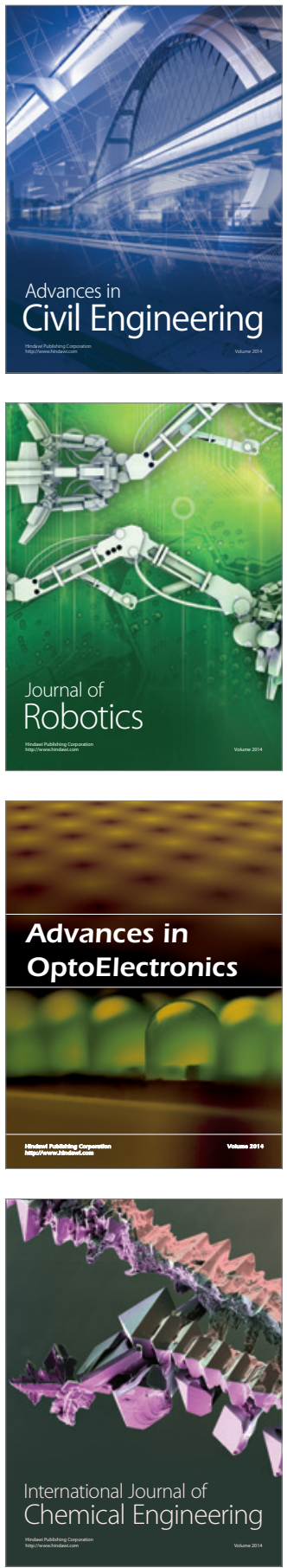

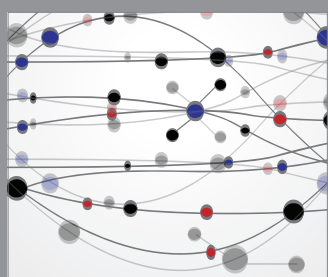

The Scientific World Journal

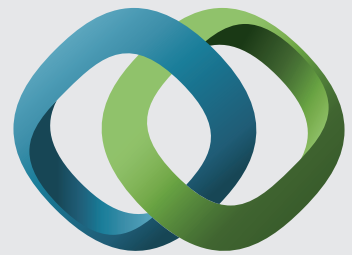

\section{Hindawi}

Submit your manuscripts at

https://www.hindawi.com
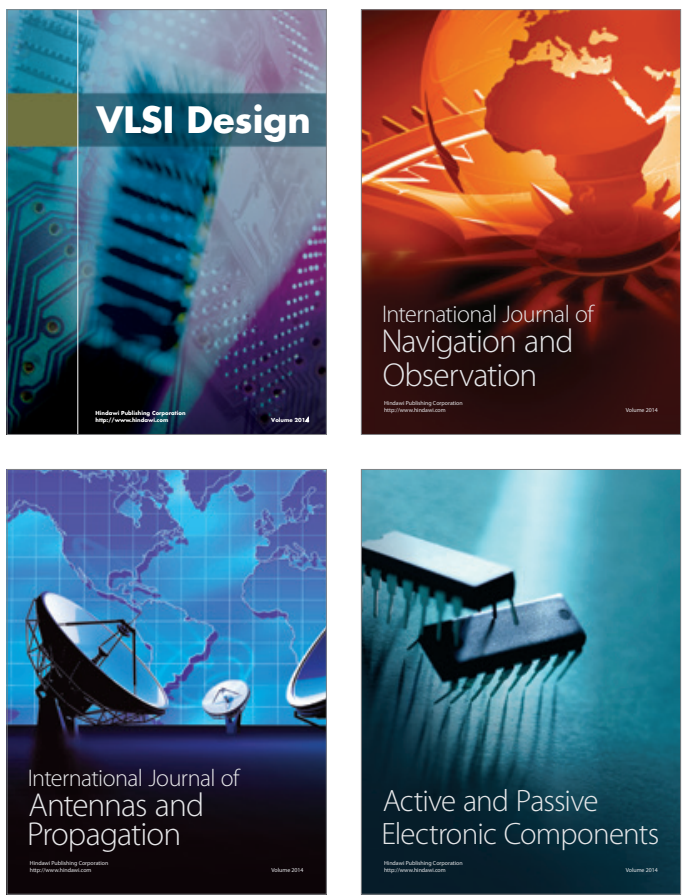
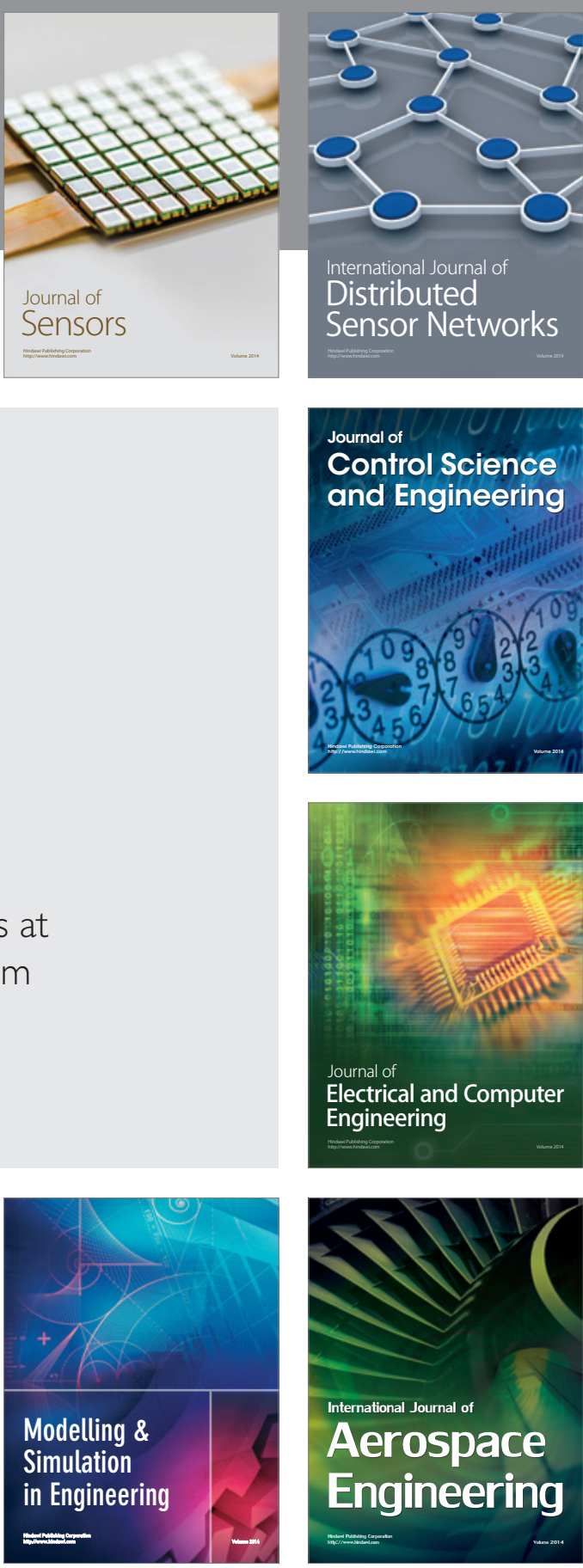

International Journal of

Distributed

Sensor Networks

$-$

Joumal of

Control Science

and Engineering
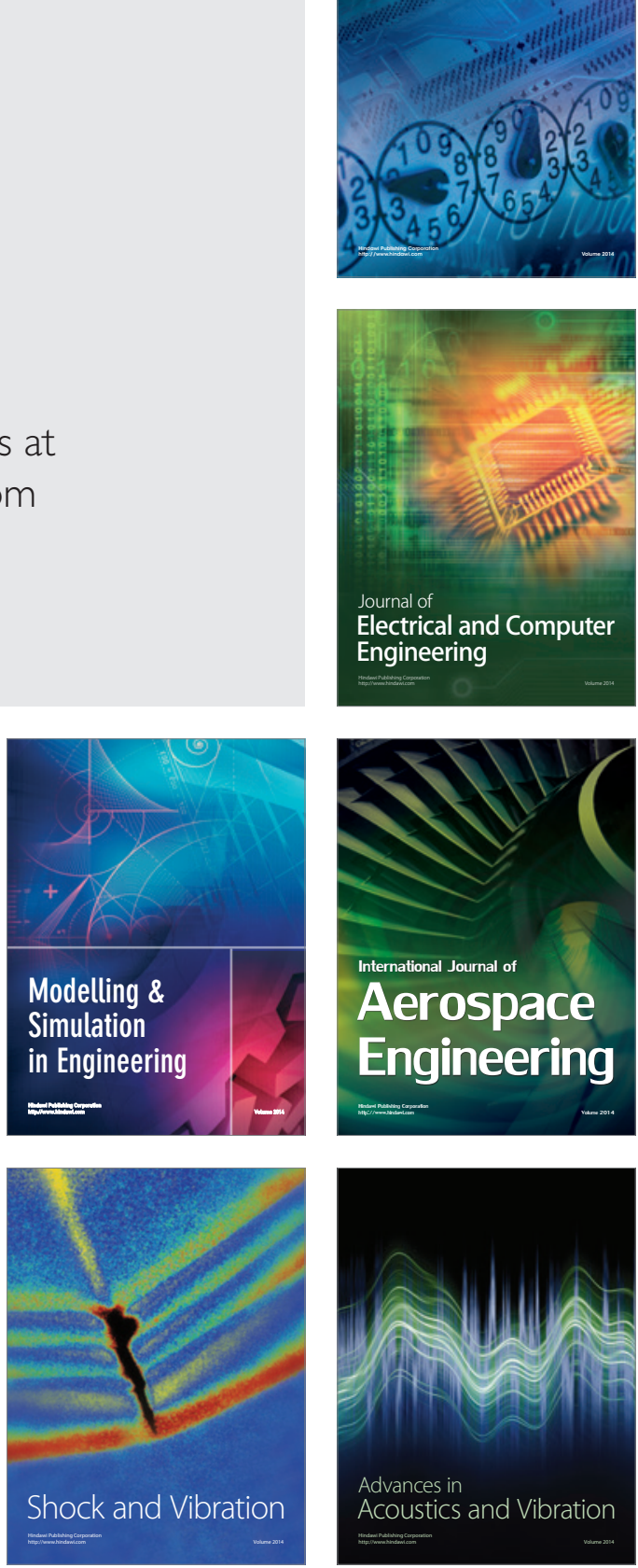